\title{
Educação parental: estudo exploratório em um grupo de dança inclusiva
}

\author{
Ana Pereira Antunes' \\ Centro de Investigação em Estudos da Criança, Universidade do Minho, Braga - Portugal \\ Centro de Competências de Artes e Humanidades, Universidade da Madeira, Funchal - Portugal \\ Joana Xavier \\ Secretaria Regional da Educação e Recursos Humanos da Madeira, Funchal - Portugal \\ Ana Tomás Almeida \\ Instituto de Educação, Universidade do Minho, Braga - Portugal
}

\begin{abstract}
Resumo: Neste trabalho avaliamos a eficácia do programa de educação parental Grupo Laços de Inclusão, implementado numa associação de arte inclusiva. Participaram no estudo II pais (três pais e oito mães) de crianças/jovens (nove com necessidades especiais e dois sem necessidades especiais) que frequentavam um grupo de dança inclusiva na Região Autónoma da Madeira (Portugal). Realizou-se uma avaliação pré-teste e pós-teste, cujos resultados parecem apontar para uma diminuição das perceções do stress global dos participantes, da exigência associada à interação com a criança e do nível de saúde dos pais que poderá interferir na relação com os filhos. Apesar de os resultados serem promissores nestas dimensões, são apresentadas algumas reflexões e limitações a ter em conta em futuras intervenções de educação parental.
\end{abstract}

Palavras-chave: educação parental; parentalidade positiva; eficácia do programa; necessidades especiais; inclusão social.

\section{PARENT EDUCATION: EXPLORATORY STUDY IN AN INCLUSIVE DANCE GROUP}

\begin{abstract}
This paper evaluates the effectiveness of a parent training program, Grupo Laços de Inclusão (Bonds of Inclusion Group), implemented in an association of inclusive art. In this study participated I I parents (three fathers and eight mothers) of children/youngsters (nine with special needs and two without disabilities) attending an inclusive dance group in Madeira Island (Portugal). It was carried out a pre-test and a post-test evaluation. The results show a decrease in the parents' perceptions of global stress, demand associated with the interaction with the child, and parents' health that could interfere with the relationship with the children. Despite the promising results, we present some reflections and limitations to take into consideration in future parent education interventions.
\end{abstract}

Keywords: parent training; positive parenting; program effectiveness; special needs; social inclusion.

${ }^{1}$ Endereço para correspondência: Ana Pereira Antunes, Universidade da Madeira, Centro de Competências de Artes e Humanidades, Edifício da Penteada, Caminho da Penteada, 9000-390, Funchal - Portugal. E-mail: aantunes@uma.pt. 
INCLUSIVA

\begin{abstract}
Resumen: El trabajo evalúa la eficacia del programa de educación parental Grupo Laços de Inclusão (Grupo Lazos de Inclusión), aplicado en una asociación de arte inclusiva, de la Región Autónoma de Madeira. Han participado en el estudio II padres (ocho madres y tres padres) de nueve niños/jóvenes con necesidades especiales y de tres niños/jóvenes sin ninguna necesidad especial. Se ha realizado una evaluación pre-test y post-test y los resultados previos indican para una disminución de la percepción: del estrés global de los participantes; de exigencias de como relacionarse con el niño; del nivel de la salud de los padres, que pueden interferir na relación con el niño. Aunque los resultados parecen ser positivos, todavia se destacan algunas limitaciones y se sacan reflexiones para futuras intervenciones de educación parental.
\end{abstract}

Palabras clave: educación parental; parentalidad positiva; eficacia del programa; necesidades especiales; inclusión social.

A família é considerada o contexto de desenvolvimento preferencial da criança, constituindo-se um espaço de interação onde são criadas oportunidades diversas de socialização, através da aprendizagem de valores, normas e comportamentos (Ceballos \& Rodrigo, 2000). No entanto, este processo não é linear nem isento de dúvidas por parte dos pais. Ao longo do exercício da parentalidade, as famílias deparam-se com desafios e inquietações diversas, sendo que estes desafios e interrogações parecem maiores quando existe uma criança com necessidades especiais (NE). Além disso, perceções de maiores níveis de stress, de adaptação e de reorganização familiar também estão presentes nestas famílias (Coutinho, 2004; Dinnebell, 1999). Estes pais e familiares manifestam mais frequentemente sentimentos de insegurança "sobre o que fazer e como o fazer" (Coutinho, 2003, p. 228). Nesse sentido, importa referir que as intervenções de educação parental com famílias de crianças com necessidades educativas especiais (NEE) ou problemas de comportamento/emocionais aparecem referenciadas, com suporte empírico, como de elevada prioridade (Mann, 2008).

A implementação de programas de educação parental surge, de uma forma geral, com o objetivo principal de reforçar os saberes parentais, pressupondo a educação parental como um processo construído conjunta e paulatinamente com os pais, não em termos instrucionais, mas de um modo preventivo e promocional (Almeida et al., 2012). De uma forma mais específica, a realização destes programas, em contextos inclusivos, revela-se essencial, na medida em que pode estimular um maior envolvimento das famílias e promover uma maior qualidade no desempenho dos seus papéis (Correia, 2003). Esta forma de intervenção junto dos pais e familiares, ao promover níveis superiores de conhecimento e capacitação, pode contribuir para o desenvolvimento de maiores perceções de competência e uma maior eficácia ao nível dos processos de participação e cooperação próprios da educação dos filhos (Coutinho, 2003).

Estes grupos de trabalho parecem constituir uma fonte de suporte emocional e de informação, mediante a partilha de experiências e situações comuns. As famílias que percebem a existência de uma rede de apoio formal e informal reagem mais adaptativamente às crises de vida (Madureira \& Leite, 2003), pois parece que esta rede funciona 
como um redutor do nível de stress e como um recurso protetor na resolução de problemas (Almeida \& Sampaio, 2007). O suporte social, formal e informal, aparece como um fator crucial a ter em conta quando se intervém ao nível da parentalidade, uma vez que esta é exercida numa teia de interações entre diversos sistemas e depende da qualidade do suporte social existente nesses mesmos sistemas, sobretudo nas famílias que experienciam condições psicossociais mais negativas (Rodrigo, 2010). A disponibilização de um suporte social adequado revela-se uma forma eficaz de prevenir a ocorrência de maus tratos porque potencia a ocorrência de mudanças nas crenças e práticas parentais que colocam a criança em risco psicossocial (Byrne, Rodrigo, \& Martin, 2012).

Atualmente, a nível europeu, assiste-se a um crescente interesse não no desenvolvimento arbitrário de programas de educação parental, mas enfatizando a sua conceção numa lógica de parentalidade positiva e baseada na investigação (Rodrigo, 2010; Rodrigo, Almeida, Spiel, \& Koops, 2012), sendo que a ênfase colocada nestes dois aspetos decorre também da Recomendação do Conselho da Europa sobre a Política de Promoção da Parentalidade Positiva (Committee of Ministers of the Council of Europe, 2006) e da importância atribuída ao papel dos pais e do Estado no desenvolvimento das crianças (ChildONEurope Secretariat, 2007). No entanto, apesar do interesse e esforço de alguns Estados Europeus em implementar ações de apoio aos pais no exercício da parentalidade, verifica-se que ainda não é possível avaliar a eficácia de algumas dessas intervenções de forma estruturada e controlada (Rodrigo et al., 2012), evidenciando a necessidade de se contemplar mecanismos de avaliação nos programas implementados, pois daí poderão fundamentar-se e desencadear-se ações mais consistentes e até mais sistémicas como, por exemplo, a regulamentação da aplicação destas formas de intervenção.

Mesmo assim, existem vários estudos que apontam para a importância da implementação destes tipos de programa, na medida em que parecem promover a otimização da informação dos pais e a melhoria das suas competências educativas (Coutinho, 2004), potenciando um desenvolvimento mais harmonioso dos filhos e com níveis superiores de autonomia (Garcia, 2007). Registam-se mudanças ao nível das teorias implícitas dos pais e no fortalecimento da rede de apoio social (Rodrigo, Byrne, Máiquez, \& Martín, 2008), sendo que, durante as sessões, os participantes parecem motivados a refletir sobre as próprias práticas educativas, apreciando a existência de um tempo e de um espaço em que podem exprimir as suas vivências e aprender também com as experiências partilhadas pelos outros elementos do grupo (Garcia, Yunes, Lucas, \& Garcia, 2010).

Verificamos que as melhorias parecem acontecer não só em termos da prática educativa, do suporte social, mas também ao nível emocional. Por exemplo, Ribeiro (2003) encontrou resultados que apontam para uma eficácia na promoção de práticas parentais democráticas, na capacidade de expressão de sentimentos positivos e na capacidade de regulação de sentimentos negativos por parte dos pais, sendo que apareceram fortalecidas as atitudes otimistas dos participantes face às dificuldades, aos outros 
e à própria vida. Importa referir que ao longo do tempo, em avaliação de follow-up, a autorregulação emocional apareceu como fator associado à intervenção.

Outros aspetos que aparecem como alvo de melhoria após a frequência de um programa de educação parental prendem-se com a ocorrência de perceções dos pais mais positivas e realistas acerca do comportamento dos filhos, maior envolvimento parental nas atividades destes, maior segurança na interação com eles e melhor relacionamento familiar, sendo que estes aspetos se traduzem numa maior satisfação com o papel parental (Biasoli-Alves, 2005). Por exemplo, num caso de crianças com problemas de comportamento, os resultados apontam para um aumento das competências sociais e educativas dos pais, influenciando de forma positiva a conduta das crianças e as interações intrafamiliares (Pinheiro, Haase, Amarante, Prette, \& Del Prette, 2006).

Em Portugal, assistimos também à realização de iniciativas de educação parental, em vários contextos de atuação, desde famílias em risco social ou famílias de crianças com NE. Por exemplo, Coutinho (2004), num programa de formação parental destinado a pais de crianças com trissomia 21, registrou um fortalecimento nos níveis de competência parental das mães. Mais recentemente, foi realizado um estudo de âmbito nacional no qual se analisaram vários programas de educação parental e a sua eficácia em diversos parâmetros, sendo que os resultados apontam para efeitos positivos, decorrentes da intervenção, junto dos pais e dos filhos, nomeadamente na redução do stress parental e na melhoria das práticas educativas, reduzindo também os comportamentos de abuso e de negligência para com as crianças, uma maior perceção do suporte social informal e pequenas melhorias no ajustamento das crianças (Almeida et al., 2012).

Nesta linha de atuação, foi desenvolvido o programa de educação parental Grupo Laços de Inclusão (GLI) e implementado na Associação dos Amigos da Arte Inclusiva Dançando com a Diferença (AAAIDD), na Região Autónoma da Madeira (RAM), sendo que este configura um contexto inclusivo por excelência, ao promover e utilizar as diferentes linguagens artísticas como elemento de inclusão social das pessoas com e sem NE (www.aaaidd.com). Neste trabalho, partindo de um estudo mais alargado (Xavier, 2011), temos como objetivo principal avaliar a eficácia do programa GLI, através da mensuração de indicadores relacionados com as características da criança e relacionados com as características dos pais e que podem estar associados a fontes de stress na relação pais-filhos.

\section{Método}

\section{Participantes}

Do grupo de 17 pais (cinco homens e doze mulheres) que integraram inicialmente o programa GLI, um elemento (homem) não participou em nenhuma sessão. Dos restantes participantes, quatro elementos (três mulheres e um homem) não permitiram a recolha de dados no pré-teste e no pós-teste. Além disso, um outro elemento (mulher) participou no pré-teste e no programa, mas não esteve disponível na fase do pós-teste. 
Face a estes constrangimentos, neste estudo contemplamos apenas o grupo constituído por pais que participaram no programa e que permitiram recolher dados para a avaliação da eficácia do programa, antes e depois da aplicação do mesmo. Assim sendo, a amostra deste estudo cinge-se a 11 pais (três pais e oito mães) de crianças/jovens (nove com necessidades especiais e dois sem necessidades especiais) que frequentaram um grupo de dança inclusiva na AAAIDD, na ilha da Madeira, em Portugal.

O grupo de participantes apresentava uma idade média de 44,3 anos ( $D P=11,94$ ), que oscilava entre os 28 e os 64 anos. Quanto ao estado civil, cinco participantes eram casados, quatro eram divorciados, um era solteiro e um vivia em união de facto. No que se refere às habilitações literárias, o grupo de pais era constituído, maioritariamente, por indivíduos com o ensino secundário (oito), sendo que três elementos apresentavam formação ao nível do terceiro ciclo do ensino básico.

\section{Instrumentos}

Para a realização desta investigação, foi necessária a implementação do programa de educação parental GLI e a aplicação, antes e depois da intervenção, do questionário Índice de Stress Parental - ISP (Abidin, 2003).

O programa de educação parental GLI pretende-se centrado na promoção de competências parentais, num grupo inclusivo, e foi construído considerando as perspetivas ecológicas e sistémicas (Coutinho, 2003; Relvas, 2006), as perspetivas narrativas e do construcionismo social (Gonçalves, 2003) e a psicologia positiva (Seligman \& Csikszentmihalyi, 2000). O GLI tem como objetivos principais: 1. desenvolver os sentimentos de pertença ao grupo e à AAAIDD e a construção de uma rede social de suporte; 2. promover a reflexão e o conhecimento da dinâmica familiar e dos respetivos estilos educativos; 3 . promover a mudança e a otimização de competências parentais; 4 . debater crenças sobre deficiência, necessidades especiais e inclusão; e 5. promover a mudança de crenças e atitudes associadas à inclusão.

O questionário ISP (Abidin, 2003) é constituído por 132 itens e as respostas são assinaladas em folha própria numa escala tipo Likert de 1 (discordo completamente) a 5 (concordo completamente) pontos. O ISP permite avaliar dois domínios considerados como fontes de stress na relação pais-filhos, ou seja, o domínio da criança (DC), referente às características da criança, e o domínio dos pais (DP), referente às características da figura parental, sendo que cada um destes domínios é constituído por diferentes subescalas, que permitem a identificação de fontes específicas de stress.

As seis subescalas do DC (distração/hiperatividade, reforço aos pais, humor, aceitação, maleabilidade de adaptação e exigência) avaliam aspetos relacionados com o temperamento da criança, bem como as perceções que os pais têm acerca do impacto que as características dos filhos têm sobre eles.

As sete subescalas do DP (sentido de competência, vinculação, restrição do papel, depressão, relação com marido/mulher, isolamento social e saúde) avaliam características específicas das figuras parentais, bem como variáveis do contexto familiar que 
influem na capacidade de responder de forma adequada às funções e aos requisitos próprios do ser pai ou mãe.

O ISP tem ainda uma escala opcional, a escala de stress de vida (SV), que permite avaliar o stress de vida situacional, stress externo à relação dos pais com a criança, que é sentido pelos mesmos, por se considerar que este pode intensificar o nível total de stress que aparece associado ao desempenho do papel parental.

Os resultados do ISP podem ser lidos de três formas: 1. considerando cada subescala de cada domínio; 2 . considerando o somatório das subescalas em cada um dos domínios; ou 3. considerando o resultado total dos dois domínios (total de stress). O resultado da escala opcional SV é independente destas três condições e o seu cálculo é realizado de forma isolada. A análise dos resultados deve ser precedida pela análise dos resultados da escala resposta defensiva, a qual indicia que os respondentes não se posicionaram de forma defensiva no preenchimento das respostas ao questionário quando o valor das respostas é igual ou superior a 24, o que permite a continuidade da análise dos dados (Abidin, 2003).

Os restantes resultados das escalas e subescalas do ISP devem ser analisados comparando-os com os percentis da amostra normativa portuguesa, sendo que são considerados normais quando o limite se situa entre o percentil 15 e o percentil 80 . Por seu lado, os resultados são considerados elevados quando se situam no percentil 85 ou acima, apontando para sistemas mãe/pai-criança em stress e, assim, em risco de desenvolverem comportamentos parentais disfuncionais ou problemas de comportamento na criança (Abidin, 2003).

\section{Procedimentos}

O projeto de investigação foi apresentado à Direcção da AAAIDD, tendo sido formalizada a aceitação e a autorização para a realização do estudo. Criadas as condições para a recolha de dados, foi marcado o pré-teste, no qual se aplicou o ISP, mediante consentimento informado e participação voluntária dos pais. De seguida, iniciou-se a aplicação do programa GLI, que decorreu ao longo de um período de quatro meses, em oito sessões quinzenais, cada uma com a duração aproximada de 60 minutos. Aquando do término do programa, foi aplicado novamente, como pós-teste, o ISP.

A correção dos dados recolhidos através do ISP foi realizada através do sistema de correção informatizado de testes CEGOC System-2010. Depois, os dados obtidos por cada sujeito, por escala e subescala do ISP, foram analisados com recurso ao programa informático IBM SPSS Statistics para Windows, versão 20.

\section{Resultados}

Nesta secção, apresentamos os resultados obtidos através da aplicação pré e pós-teste do questionário ISP (Abidin, 2003), em cada uma das suas escalas e subescalas. Apesar da variabilidade dos sujeitos, como se pode constatar na caracterização dos participantes, a opção seguida neste trabalho foi a de considerar o grupo de participantes 
como uma primeira abordagem, a partir da qual se poderão equacionar análises de perfis individuais em abordagens posteriores. Dado o número de participantes no estudo ser bastante reduzido (11 elementos), optou-se por uma análise estatística, para comparação de resultados, através de testes não paramétricos, ou seja, o teste não paramétrico de Wilcoxon.

Antes de procedermos à apresentação dos dados nas várias escalas (Tabela 1), importa começar a análise pelos resultados da escala resposta defensiva, procurando avaliar se os participantes responderam ao questionário de forma defensiva.

\section{Tabela I. Resultados dos pais nas escalas do ISP no pré-teste e pós-teste}

\begin{tabular}{llcccccc}
\hline & & & & & & \multicolumn{2}{c}{ Teste de Wilcoxon } \\
\cline { 7 - 8 } Escala & Momento & M & DP & Mín. & Máx. & $Z$ & Z \\
\hline Resposta defensiva & Pré-teste & 32,7 & 4,80 & 26 & 40 & $-0,527$ & 0,797 \\
& Pós-teste & 32,3 & 5,22 & 22 & 40 & & \\
\hline Total de stress & Pré-teste & 220,2 & 28,88 & 157 & 261 & $-1,956$ & 0,050 \\
& Pós-teste & 205,2 & 22,96 & 163 & 236 & & \\
\hline Stress de vida & Pré-teste & 19,0 & 15,70 & 0 & 38 & $-0,210$ & 0,833 \\
& Pós-teste & 19,1 & 17,36 & 0 & 45 & & \\
\hline Domínio da criança & Pré-teste & 113,3 & 20,64 & 76 & 147 & $-2,192$ & 0,028 \\
& Pós-teste & 101,9 & 13,35 & 76 & 126 & & \\
\hline Domínio dos pais & Pré-teste & 116,0 & 13,36 & 95 & 146 & $-1,172$ & 0,241 \\
& Pós-teste & 112,4 & 18,91 & 87 & 155 & & \\
\hline
\end{tabular}

Fonte: Elaborada pelas autoras.

Ao consultarmos a Tabela 1, verificamos que o valor médio da resposta defensiva, em ambos os momentos de avaliação $\left(\mathrm{M}_{\text {pré }}=32,7\right.$ e $\left.\mathrm{M}_{\text {pós }}=32,3\right)$, se encontra acima do valor 24, que representa o ponto de corte a ter em conta na indicação de respostas defensivas, ou seja, valores iguais ou superiores a 24 não indiciam uma atitude defensiva por parte dos participantes pelo que podemos continuar com a análise das respostas (Abidin, 2003).

Ao continuarmos a análise da Tabela 1, constata-se que, para a escala total de stress, entre o pré-teste e o pós-teste, ocorreu uma diminuição no valor das médias obtidas $\left(M_{\text {pré }}=220,2\right.$ e $\left.M_{\text {pós }}=205,2\right)$, sendo que esta diferença se revela quase estatisticamente significativa $(p=0,05)$. Estes valores médios não indiciam perceções globais de stress preocupantes, uma vez que se situam, aproximadamente, no percentil 55 no pré-teste e no percentil 35 no pós-teste. No entanto, para a escala stress de vida, os valores médios ( $M_{\text {pré }}=19,0$ e $\left.M_{\text {pós }}=19,1\right)$ são muito próximos e não se apresentam 
estatisticamente significativos, mas estes valores aproximam-se do percentil 90, o que significa que estes pais vivenciam um stress situacional acentuado.

No que se refere à escala domínio da criança, verificamos, na mesma tabela, uma diminuição no valor das médias obtidas no valor global do $D C\left(M_{\text {pré}}=113,3\right.$ e $\left.M_{\text {pós }}=101,9\right)$, entre o pré-teste e o pós-teste, revelando-se uma diferença estatisticamente significativa $(p<0,05)$. Estes resultados médios correspondem no pré-teste ao percentil 75 e no pós-teste ao percentil 50, revelando mais uma vez que os resultados médios não indiciam um grupo que percebe as características das crianças como geradoras de stress elevado. Ao analisar-se o domínio dos pais, constatamos que ocorreu uma diminuição no valor das médias obtidas entre o pré e o pós-teste $\left(M_{\text {pré }}=116,0\right.$ e $\left.M_{\text {pós }}=112,4\right)$, sendo que essas diferenças não apresentam significância estatística e oscilam, aproximadamente, entre o percentil 45 e o percentil 40, não indiciando perceções da figura parental apontando para disfuncionalidade na relação com os filhos.

Depois de uma apreciação global no DC, vamos proceder a uma análise dos resultados nas subescalas que compõem este domínio, em cada um dos momentos de avaliação (Tabela 2).

\section{Tabela 2. Resultados dos pais nas subescalas do DC no pré-teste e pós-teste}

\begin{tabular}{llcccccc} 
& & & & & \multicolumn{2}{c}{ Teste de Wilcoxon } \\
\cline { 6 - 8 } Subescala & Momento & $M$ & DP & Mín. & Máx. & Z & P \\
\hline D/H & Pré-teste & 24,4 & 5,24 & 16 & 34 & $-1,533$ & 0,125 \\
& Pós-teste & 22,3 & 3,50 & 15 & 26 & & \\
\hline Reforço aos pais & Pré-teste & 9,4 & 1,86 & 6 & 11 & $-1,131$ & 0,258 \\
& Pós-teste & 8,6 & 2,51 & 6 & 13 & & \\
\hline Humor & Pré-teste & 10,6 & 4,23 & 4 & 17 & $-1,784$ & 0,074 \\
& Pós-teste & 8,7 & 2,76 & 4 & 14 & & \\
\hline Aceitação & Pré-teste & 20,6 & 5,15 & 12 & 29 & $-1,421$ & 0,155 \\
& Pós-teste & 18,6 & 2,66 & 14 & 22 & & \\
\hline MA & Pré-teste & 29,1 & 7,41 & 14 & 45 & $-1,876$ & 0,061 \\
& Pós-teste & 26,6 & 6,30 & 14 & 39 & & \\
\hline Exigência & Pré-teste & 19,2 & 5,08 & 9 & 26 & $-1,876$ & 0,027 \\
& Pós-teste & 16,9 & 3,75 & 9 & 23 & & \\
\hline
\end{tabular}

D/H: distração/hiperatividade; MA: maleabilidade de adaptação.

Fonte: Elaborada pelas autoras.

Ao consultarmos os resultados na Tabela 2, verificamos, de uma forma geral, uma diminuição do valor médio obtido pelos pais no pós-teste para todas as subescalas do 
DC, sendo que todos eles se encontram no leque de percentis considerados normais. No entanto, para a subescala exigência $\left(M_{\text {pré }}=19,2\right.$ e $\left.M_{\text {pós }}=16,9\right)$ essa diferença revela-se estatisticamente significativa $(p<0,05)$.

Da mesma forma, depois da apreciação global no DP, apresentamos os resultados nas subescalas que compõem este domínio, nos dois momentos de avaliação (Tabela 3).

\section{Tabela 3. Resultados dos pais nas subescalas do DP no pré-teste e pós-teste}

\begin{tabular}{llccccccc} 
& & & & & & \multicolumn{2}{c}{ Teste de Wilcoxon } \\
\cline { 7 - 8 } Subescala & Momento & M & DP & Mín. & Máx. & Z & P \\
\hline SC & Pré-teste & 26,5 & 5,73 & 19 & 37 & $-1,403$ & 0,161 \\
& Pós-teste & 27,7 & 6,75 & 19 & 44 & & \\
\hline Vinculação & Pré-teste & 11,7 & 3,55 & 7 & 18 & $-0,647$ & 0,518 \\
& Pós-teste & 11,3 & 3,41 & 7 & 20 & & \\
\hline Restrição do papel & Pré-teste & 14,8 & 3,22 & 10 & 20 & $-1,207$ & 0,228 \\
& Pós-teste & 14,2 & 2,99 & 8 & 18 & & \\
\hline Depressão & Pré-teste & 22,6 & 5,15 & 16 & 34 & $-0,981$ & 0,326 \\
& Pós-teste & 21,0 & 2,93 & 17 & 27 & & \\
\hline Relação M/M & Pré-teste & 16,2 & 5,44 & 10 & 29 & $-0,962$ & 0,336 \\
& Pós-teste & 15,7 & 6,13 & 8 & 29 & & \\
\hline Isolamento social & Pré-teste & 13,2 & 3,46 & 7 & 17 & $-0,960$ & 0,337 \\
& Pós-teste & 12,5 & 3,66 & 6 & 18 & & \\
\hline Saúde & Pré-teste & 12,1 & 3,56 & 8 & 21 & $-2,099$ & 0,036 \\
& Pós-teste & 10,0 & 2,24 & 7 & 14 & & \\
\hline
\end{tabular}

SC: sentido de competência; relação $M / M$ : relação marido/mulher.

Fonte: Elaborada pelas autoras.

Ao analisarmos a Tabela 3, constatamos que, de uma forma geral, também ocorreu uma diminuição no valor das médias obtidas entre o pré e o pós-teste, sendo que essas diferenças não apresentam significância estatística e correspondem a percentis considerados normais. Como exceção temos os resultados na subescala saúde $\left(\mathrm{M}_{\text {pré }}=12,1 \mathrm{e}\right.$ $\left.M_{\text {pós }}=10,0\right)$, em que a diferença é estatisticamente significativa $(p<0,05)$. Curiosamente, na subescala sentido de competência, encontramos um ligeiro aumento dos valores médios $\left(M_{\text {pré }}=26,5\right.$ e $\left.M_{\text {pós }}=27,7\right)$, mas que não revela significância estatística.

\section{Discussão}

Os resultados encontrados apontam para uma eficácia do programa GLI na diminuição do stress global experienciado pelos pais e na diminuição das perceções das 
características das crianças indutoras de stress. De uma forma mais específica, registra-se uma diminuição do nível de exigência associado à interação com a criança e uma diminuição da perceção da deterioração do nível de saúde dos pais, que poderá interferir na relação com os filhos.

Importa referir que a diminuição dos valores médios encontrados e os restantes valores nas outras subescalas encontram-se, em termos médios, dentro do leque de percentis considerados normais (entre o percentil 15 e o percentil 80), o que significa que este grupo de pais não parece apresentar características de disfuncionalidade. A exceção a este facto acontece com a subescala stress de vida, que avalia o stress de vida situacional e que aparece com valores bastante elevados em ambos os momentos de avaliação (próximos do percentil 90), o que poderá indiciar perceções de acontecimentos ou circunstâncias stressantes externas à relação pai/mãe-criança, que não se alteraram com a frequência do programa. Contudo, ao nível do stress global e das características da criança como geradoras de stress, houve uma alteração das perceções dos pais, o que nos leva a supor que a participação no programa pode ter ajudado estes pais a relativizarem alguns aspetos sobre a parentalidade e a iniciarem um processo de modificação de crenças pessoais como, aliás, se tem verificado noutros estudos (Almeida et al., 2012; Biasoli-Alves, 2005; Garcia et al., 2010; Ribeiro, 2003; Rodrigo et al., 2008). Aliás, verificamos um aumento dos valores médios na subescala sentido de competência que, apesar de não ser estatisticamente significativo, reforça a ideia de empoderamento e mudança das atitudes parentais.

Se relembrarmos que o grupo de participantes é constituído, maioritariamente, por pais de crianças com NE, referenciados como alvos prioritários deste tipo de intervenção (Correia, 2003; Mann, 2008), parece-nos que os resultados encontrados reforçam o sentido de eficácia do programa e a relevância de se potenciar e continuar a aposta na educação parental, em geral e em contextos inclusivos. Parece-nos que, a partir destes dados tomando o grupo como referência de análise, seria interessante dar continuidade a este estudo e procurar analisar os perfis individuais de cada um dos participantes e verificar que fatores poderão estar associados ou justificar os valores encontrados, sejam eles considerados normativos ou indiciadores de stress parental, para cada um dos momentos de avaliação. De uma forma mais específica, tendo sido o programa desenvolvido no âmbito de atuação da AAAIDD, importaria assumir esta forma de intervenção e divulgar os dados junto dos pais e dos elementos da equipa técnica da associação, como já aconteceu, mas também individualmente para que cada pai e cada mãe pudessem refletir sobre o processo individual de participação no GLI.

Outro aspeto importante remete-nos para o suporte social que os participantes sentem através da participação nestes programas (Almeida \& Sampaio, 2007; Madureira \& Leite, 2003; Rodrigo, 2010), facto que também se registou neste grupo, através de diversas verbalizações, ao longo das sessões, revelando perceções do grupo como facilitador de partilha, suporte e aprendizagem (Xavier, 2011). Mais uma vez, estes aspetos reforçam a importância que a AAAIDD pode ter no suporte de pais de crianças e jovens com NE, mas também no combate à discriminação e na aceitação da diversi- 
dade, através do debate de crenças que proporciona e da atuação social que vai conseguindo através dos espetáculos de dança inclusiva. No entanto, apesar da motivação dos pais para participarem no programa, verificaram-se algumas oscilações, por parte de alguns elementos, na assiduidade às sessões, sendo que esta dificuldade se tem verificado noutros estudos (Axford, Lehtonen, Kaoukji, Tobin, \& Berry, 2012). Efetivamente só houve desistência de um elemento na participação no programa, mas importa referir que, mesmo tendo sido um grupo muito motivado para a intervenção, a não colaboração de alguns pais na avaliação do GLI poderá justificar-se pelo receio à exposição, resultado do peso do estigma da diferença e da exclusão social, preferindo alguns deles permanecer invisíveis. Este aspeto constitui, em nosso entender, uma limitação do estudo, e, em futuras intervenções de educação parental na AAAIDD, parece-nos que importaria assegurar algumas condições com o intuito de prevenir a falta de compromisso e assiduidade dos participantes. Assim, deveriam rever-se alguns aspetos ao nível da implementação do GLI como, por exemplo, reduzir a periodicidade intervalar das sessões, aumentar a duração de cada sessão, aumentar o número de sessões e selecionar os pais em função da faixa etária dos filhos, separando a infância da adolescência. Além disso, deveria ser reforçada, junto dos pais, uma maior sensibilização para a assiduidade e para a importância da participação nos momentos de avaliação. Pensamos que o número de participantes em cada grupo também deve ser cuidado e reforçada a ideia de compromisso assumido. Em estudos futuros, em termos metodológicos, a replicação do programa junto de outros pais e a existência de um grupo de controle poderiam implicar mais contributos à avaliação da eficácia do GLI e perceber com mais detalhes os ganhos dos pais com a participação no programa.

\section{Conclusão}

A presente investigação traduz também o reconhecimento da AAAIDD da relevância que deve ser dada à educação parental, tendo-a já assegurada nas suas vertentes de atuação, mas carecendo, no momento do estudo, de avaliação sistematizada. Pois bem, parece-nos que estes resultados, ainda que obtidos num pequeno grupo de participantes, constituem um contributo promissor à implementação e eficácia da educação parental em contextos inclusivos. Esperamos, ainda, que o contributo do estudo se estenda à crescente reflexão sobre a inclusão social e à concretização de uma sociedade cada vez mais inclusiva e aberta à diversidade.

\section{Referências}

Abidin, R. (2003). Índice de stress parental (PSI): parenting stress index (S. Santos, Adap. port.). Lisboa: Cegoc-TEA.

Almeida, A., Abreu-Lima, I., Cruz, O., Gaspar, M. F., Brandão, T., Alarcão, M., Santos, M. R., \& Machado, J. C. (2012). Parent education interventions: results from a national study in Portugal. European Journal of Developmental Psychology, 9(1), 135-149. 
Almeida, T., \& Sampaio, F. (2007). Stress e suporte social em familiares de pessoas com paralisia cerebral. Psicologia, Saúde e Doenças, 8(1), 145-151.

Axford, N., Lehtonen, M., Kaoukji, D., Tobin, K., \& Berry, V. (2012). Engaging parents in parenting programs: lessons from research and practice. Children and Youth Services Review, 34, 2061-2071.

Biasoli-Alves, Z. M. M. (2005). Orientação de pais: partilhar conhecimentos sobre desenvolvimento e práticas de educação como estratégia de intervenção. Texto \& Contexto Enfermagem, 14, 64-70.

Byrne, S., Rodrigo, M. J., \& Martin, J. C. (2012). Influence of form and timing of social support on parental outcomes of a child-malteatment prevention program. Children and Youth Services Review, 34, 2495-2503.

Ceballos, E., \& Rodrigo, M. J. (2000). Las metas y estratégias de socialización entre padres e hijos. In M. J. Rodrigo \& J. Palácios (Eds.). Familia y desarrollo humano (pp. 225-243). Madrid: Alianza Editorial.

ChildONEurope Secretariat (2007). Survey on the role of parents and the support from the governments in the EU. Florence: Instituto degli Innocenti. Recuperado em 25 fevereiro, 2013, de http://www.childoneurope.org/issues/support_family/ reportSurveyRoleParents.pdf.

Committee of Ministers of the Council of Europe (2006). Recommendation 19. Recuperado em 25 fevereiro, 2013, de https://wcd.coe.int/ViewDoc.jsp?id=1073507.

Correia, M. (2003). Inclusão e necessidades educativas especiais. Um guia para educadores e professores. Porto: Porto.

Coutinho, M. (2003). Formação parental: avaliação do impacte na família. Psicologia, 17(1), 227-244.

Coutinho, M. (2004). Apoio à família e formação parental. Análise Psicológica, 22(1), 55-64.

Dinnebell, L. (1999). Defining parent education in early intervenion. Topics in Early Childhood Special Education, 19(3), 161-164.

Garcia, N. M. (2007). Educação nas famílias de pescadores artesanais: transmissão geracional e processos de resiliência. Dissertação de mestrado, Universidade Federal do Rio Grande, Rio Grande, RS, Brasil.

Garcia, N. M., Yunes, M. A. M., Lucas, L. M. B., \& Garcia, S. F. (2010). Programas de educação familiar e suas contribuições para o exercício da parentalidade e cidadania. In C. S. Hutz (Org.). Avanços em psicologia comunitária e intervenções psicossociais (pp. 165-189). São Paulo: Casa do Psicólogo.

Gonçalves, M. (2003). Psicoterapia, uma arte retórica: contributos das terapias narrativas. Coimbra: Quarteto. 
Madureira, I., \& Leite, T. (2003). Necessidades educativas especiais. Lisboa: Universidade Aberta.

Mann, B. (2008). What works for whom? Promising practices in parenting education. Ottawa: Canadian Association of Family Resource Programs.

Pinheiro, M. I., Haase, V. G., Amarante, C. L., Prette, A. D., \& Del Prette, Z. A. (2006). Treinamento de habilidades sociais e educativas para pais de crianças com problemas de comportamento. Psicologia: Reflexão e Crítica, 19(3), 407-414.

Relvas, A. P. (2006). O ciclo vital da família: perspectiva sistémica (4a ed.). Porto: Edições Afrontamento.

Ribeiro, M. (2003). Ser família. Construção, implementação e avaliação de um programa de educação parental. Dissertação de mestrado, Universidade do Minho, Braga, Portugal.

Rodrigo, M. J. (2010). Promoting positive parenting in Europe: new challanges for the European Society for Development Psychology. European Journal of Developmental Psychology, 7(3), 281-294.

Rodrigo, M. J., Almeida, A., Spiel, C., \& Koops, W. (2012). Introduction: evidencebased parent education programs to promote positive parenting. European Journal of Developmental Psychology, 9(1), 2-10.

Rodrigo, M. J., Byrne, S., Máiquez, M. L., \& Martín, J. C. (2008). Preservación familiar: resultados del programa de "Apoyo Personal y Familiar" para padres en riesgo psicosocial. In J. A. González-Pienda \& J. C. Pérez (Orgs.). Atas do V Congreso Internacional de Psicología y Educación: los retos del futuro (p. 101). Universidade de Oviedo.

Seligman, M. E. P., \& Csikszentmihalyi, M. (2000). Positive psychology: an introduction. American Psychologist, 55(1), 5-14.

Xavier, J. O. (2011). Educação parental: o significado da participação no Grupo Laços de Inclusão. Dissertação de mestrado, Universidade da Madeira, Funchal, Portugal.

Submissão: 15.10.2013

Aceitação: 22.9 .2014 